\title{
First outbreak reported caused by Erysipelothrix species strain 2 in turkeys from poultry-producing farms in Brazil
}

\author{
Patrícia G. Hoepers ${ }^{1}$ - Thais F. M. dos Reis ${ }^{1}$ - Eliane P. Mendonça ${ }^{2}$ - Daise A. Rossi ${ }^{1}$ • Priscilla K. Koerich ${ }^{3}$. \\ Tiago V. J. França ${ }^{4}$. João P. Zuffo ${ }^{5}$ Edson C. V. Junior ${ }^{1} \cdot$ Belchiolina B. Fonseca $^{1}$ (D)
}

Received: 19 June 2019 / Accepted: 25 July 2019/Published online: 13 August 2019

(C) Università degli studi di Milano 2019

\begin{abstract}
Purpose The aims of this study were to report the first isolation of Erysipelothrix sp. strain 2 (ES2) associated with clinical signs of diseases as well as mortality in turkeys and identify the antimicrobial resistance of the isolates.

Methods We evaluated 118 farms for bacteriological analysis and TaqMan real-time PCR to identify the microorganism in different organs. After this, we made the epidemiological analysis between the positive flocks and the mortality mean. We performed the sequencing of the 16S rRNA region and the assessment of antimicrobial resistance.

Results We have identified $18(15.25 \%)$ as ES2-positive flocks, without any other species from the same genus being found. After analysing the organ samples, we found liver as the organ of choice for the isolation of the ES2. The sequencing of $16 \mathrm{~S}$ rRNA region of ES2 identified high homology with E. tonsillarum and E. rhusiopathiae, suggesting that it is not the best-suited target to identify this species. We have found a positive association between isolation of the bacteria in organs and flocks' mortality. Positive flocks had a mortality mean rate of $6.87 \%$, which is significantly greater than $3.76 \%$ in negative flocks. Ill turkeys had gross lesions of generalized septicaemia. The bacterial isolates showed high resistance to fosfomycin and trimethoprim/sulfamethoxazole and sensibility to norfloxacin, amoxicillin and lincomycin/spectinomycin.

Conclusion This is the first study in the world that addressed ES2 as the causative agent of erysipelas in turkey.
\end{abstract}

Keywords Poultry $\cdot$ Erysipelas $\cdot$ Septicaemia $\cdot 16 \mathrm{~S}$ rRNA region sequencing $\cdot$ Antimicrobial resistance

Electronic supplementary material The online version of this article (https://doi.org/10.1007/s13213-019-01505-3) contains supplementary material, which is available to authorized users.

Belchiolina B. Fonseca

biafonseca@ufu.com.br

Patrícia G. Hoepers

patriciag.hoepers@gmail.com

Thais F. M. dos Reis

thais.fernanda@ufu.br

Eliane P. Mendonça

eliane_vet@yahoo.com.br

Daise A. Rossi

daise.rossi@ufu.br

Priscilla K. Koerich

prikoerich@hotmail.com

Tiago V. J. França

prikoerich@hotmail.com
João P. Zuffo

Jpzuffo@yahoo.com

Edson C. V. Junior

edson2campos@hotmail.com

1 Universidade Federal de Uberlândia, Uberlândia, Minas Gerais 38402-018, Brazil

2 Universidade de Uberaba (UNIUBE), Uberaba, MG 38055-500, Brazil

3 Universidade Federal do Rio Grande do Sul, Porto Alegre, Brazil

4 BRF Brasil Food, São Paulo, Brazil

5 UDESC-Universidade do estado de Santa Catarina, Cedima, Centro de Diagnóstico de Microbiologia Animal, Florianopolis, SC 88035-901, Brazil 


\section{Findings}

Erysipelothrix is a genus of bacteria that until very recently was thought to comprise only the Erysipelothrix rhusiopathiae species, shown to be untrue due to DNADNA hybridization advancements (Takahashi et al. 1992). Multilocus enzyme electrophoresis and restriction fragment length polymorphism methods (Chooromoney et al. 1994; Ahrné et al. 1995) were important to separate Erysipelothrix into six different species, E. rhusiopathiae, E. tonsillarum, Erysipelothrix sp. strain 1 (ES1), Erysipelothrix sp. strain 2 (ES2), E. inopinata and E. larvae (Takahashi et al. 1992; Takeshi et al. 1999; Verbarg 2004; Bang et al. 2016). The infection prevalence of Erysipelothrix occurs worldwide and affects a wide variety of invertebrate and vertebrate groups, such as humans. In addition, Erysipelothrix rhusiopathiae is the etiological agent of turkey erysipelas, being characterized by an acute and fulminating infection, which in turns may affect the fertility of the male turkey. Correctly identifying the disease is then a key factor for controlling erysipelas to avoid having downgrading caused by septicaemia lesions (Bricker and Saif, 2013).

In Brazil, there has been an outbreak in 70-day-old broiler male turkeys, characterized by septicaemia and high mortality. The aim of this study was to report the first isolation of ES2 associated with clinical signs of diseases as well as mortality in turkeys, as well as to identify the antimicrobial resistance of the isolates.

From January 2014 to February 2015, we analysed pooled organ samples from 167 flocks of Nicholas male broiler turkeys. The surveyed farms were in Minas Gerais state in Brazil and had a partnership with a large poultry and swineproducing company. The flocks had from 4200 to 23,605 birds and the samples were collected from individuals from a day old to 170 days. Necropsy and sample collection were done as a routine practice in the company to address the mortality cause; 2-3 individuals were necropsied in each flock.

The necropsied birds of this work were naturally and recently dead (up to $1 \mathrm{~h}$ after death). The coelomic cavity was accessed with clean scissors, having the following organs sampled: trachea, lungs, heart, kidneys, spleen, liver, joints and intestines. The same organs from different birds within the same flock were pooled together in proper packing (Nascoß). The samples were kept refrigerated with ice packs until analysis in the laboratory of Animal Health (UberlândiaMG). The exterior surface from each organ was heat-sterilized, and the internal part of the organ was inoculated by inprint on sheep blood and MacConkey agar (Oxoid®) and then incubated aerobically at $37{ }^{\circ} \mathrm{C}$ from 18 to $24 \mathrm{~h}$. After incubation, colonies were routinely analysed by biochemical tests for the following agents: Pasteurella multocida, Escherichia coli, Erysipelothrix rhusiopathiae, Salmonella spp., Streptococcus spp., Staphylococcus spp. and Aspergillus spp. (Zavalla et al. 2008). In order to identify as
Avian Pathogenic Escherichia coli (APEC), we performed Taqman PCR using a commercial kit (Simbios Biotecnologia). Positive isolates to four or five of the following virulence-associated genes (VAG) were considered APEC according to Ikuta et al. (2014). Mycoplasma gallisepticum and Mycoplasma synoviae were analysed with a commercial real-time PCR (Simbios ${ }^{\circledR}$ ), following the protocol described by the manufacturer.

Erysipelothrix spp. colonies isolated on sheep blood agar were selected to TaqMan real-time PCR. We performed TaqMan real-time PCR for differentiation between E. rhusiopathiae, E. tonsillarum and ES2 as previously described by Pal et al. (2010) with few modifications. Ten typical colonies of each isolate were harvested and suspended in ultrapure water (Invitrogen ${ }^{\circledR}$ ) and the suspension was then used for DNA extraction using the Blood and Tissue kit (Qiagen ${ }^{\circledR}$ ), following the manufacturer's instructions. For each test, we used positive controls of E. rhusiopathiae and E. tonsillarum isolated from swine, kindly provided by Professor Dr. David Emílio Santos Neves de Barcellos (Universidade Federal do Rio Grande do Sul-UFRGS, Brazil). The amplification and identification were performed in three reactions of TaqMan real-time PCR, one reaction for each species with commercial master mix (TaqMan® Universal PCR master mix, Applied Biosystems $\left.{ }^{\circledR}\right)$. The reactions were carried out in a StepOne Real-Time PCR system (Applied Biosystems ${ }^{\circledR}$ ) with $55{ }^{\circ} \mathrm{C}$ the annealing temperature. The results were analysed with StepOne Plus (version 2.1) software. We considered samples to be positive when the $\mathrm{Ct}$ value was $\leq 35$.

ES2 was isolated and confirmed by PCR in 23 flocks, all flocks were older than 70 days. In order to make the data and mortality analysis, we excluded the flocks that were younger than 70 days when the samples were collected. The mortality was due to environmental causes such as excessive heat; significative differences in management and feed consumption were excluded. Besides, positive flocks for the following pathogens were excluded from the analysis: APEC, Mycoplasma galliepticum, Mycoplasma sinoviae, Pasteurella multocida and Salmonella spp. Thus, 118 flocks were selected (960 organ samples), being 18 positives to ES2, two were collected twice in their lifetime (160 samples) and 100 negative to ES2 and the pathogens cited above (800 samples). Five ES2 positive flocks were excluded due to concomitant positivity to APEC (three flocks) and Pastaurella multocida type D (two flocks).

We performed an epidemiological study by association analyses with the selected 18 positive flocks and 10 negative control flocks ( 80 samples). We randomly selected 10 flocks from 85 flocks that had negative tested organ samples to the pathogens cited above. We recorded the weekly mortality of the selected lots from 70 weeks of age until the slaughter age.

Among the studied isolates, six strains identified as ES2 by the TaqMan real-time PCR technique were randomly selected, and their total genomic DNA samples were sent to 
Macrogen ${ }^{\circledR}$ (Korea), so that the 16S rRNA gene could be amplified using the primers $27 \mathrm{~F}$ (5'-AGAGTT TGATCMTGGCTCAG-3) and 1492R (5'-CGGT TACCTTGTTACGACTT-3). The amplified product was then used to sequence reactions using primers $785 \mathrm{~F}$ (5'-GGAT TAGATACCCTGGTA-3) and 907R (5'-CCGT CAATTCCTTTRAGTTT-3). The DNA sequence was compared with data stored in the GenBank database, available on the National Center for Biotechnology Information websiteNCBI (www.ncbi.nlm.nih.gov).

We randomly selected $28 \mathrm{ES} 2$ isolates (at least one isolate per flock) to address antibiotic resistance using the disc diffusion test, following the protocol described by the Clinical and Laboratory Standards Institute guidelines, using Staphylococcus aureus (ATCC 2923) as a control (Cohen et al. 2008). The tested antimicrobial agents were as follows: norfloxacin $(10 \mu \mathrm{g})$, amoxicillin $(10 \mu \mathrm{g})$, enrofloxacin $(5 \mu \mathrm{g})$, ceftiofur $(30 \mu \mathrm{g})$, tetracycline $(30 \mu \mathrm{g})$, neomycin $(30 \mu \mathrm{g})$, trimethoprim/sulfamethoxazole $(1.25 \mu \mathrm{g} / 23.75 \mu \mathrm{g})$, lincomycin-spectinomycin $(109 \mu \mathrm{g})$, fosfomycin $(50 \mu \mathrm{g})$, apramycin $(15 \mu \mathrm{g})$ and oxytetracycline $(30 \mu \mathrm{g})$. All used discs for the diffusion test analysis belonged to the Oxoid®'s brand. We defined isolates as multidrug resistant when they showed resistance to $\geq 3$ classes of antimicrobial agents (Cohen et al. 2008).

We performed an epidemiological analysis by measuring the association between the average number of mortality and positivity for ES2 using the Fisher's exact test followed by an analysis of differences between proportions $(p<0.05)$ and the odds ratio calculation (OR). The prevalence and antimicrobial resistance of ES2 were analysed with descriptive statistics. We used GraphPad Prism (version 7.04).

We isolated and confirmed through real-time PCR the presence of ES2 in $15.25 \%(18 / 118)$ of the selected flocks. In the 18 positive flocks, within 160 samples, 58 tested positive to ES2. We compared the mortality mean from the 18 ES2positive flocks with 10 randomly chosen flocks (control group) and found a positive association between mortality rate and ES2 incidence. The mortality mean rates for positive and control group were $6.87 \%$ and $3.76 \%$, respectively, with positive association (Table 1). The OR results showed that there are 1.901 times more chances of ES2 positive flocks to die than negative flocks.

The mainly observed gross lesions were friable, enlarged and in some cases mottled liver, spleen and kidneys. In some

Table 1 ES2 isolates and positive association with bird's mean mortality

\begin{tabular}{lll}
\hline & Dead turkeys & Live turkeys \\
\hline Pos turkeys & 694 & 10,105 \\
Abs turkeys & 397 & 10,553 \\
\hline
\end{tabular}

Pos ES2-positive individuals, Abs ES2-negative individuals. $p<0.0001$ flocks, turkeys also had slight catarrhal enteritis. Clinical signs and gross lesions found in the present study for ES2-positive flocks are in accordance with the reported outbreaks of $E$. rhusiopathiae in turkeys. Erysipelas can cause sudden increase in the mortality rate of turkey flocks, increasing in the following days to the rest of the individuals of the same flock. Even though the mortality rate for outbreaks of E. rhusiopathiae in poultry has already been previously reported (Bricker and Saif, 2013), this is the first study of mortality caused by ES2. In order to guarantee the animal welfare and prevent economic losses, several flocks received broadspectrum antibiotic treatment in drinking water after the onset of the mortality. Therefore, the mortality rate showed in this study $(6.87 \%)$ could have been higher, since the mortality probably was decreased with the usage of antibiotics.

Although E. rhusiopathiae has been considered pathogenic for turkeys at any age (Bricker and Saif, 2013; Schmitt et al. 2014) in our study, the epidemiological analysis showed that ES2 was only clinically diagnosed after 70 days of bird's age. On average, the birds were 112 days old at the onset of the disease.

We have primarily isolated ES2 from samples from the following organs ES2 was isolated from samples of liver $(88.9 \%, 16 / 18)$, spleen $(83.3 \%, 15 / 18)$, heart $(55.55 \%, 10 /$ $18)$, lung $(38.9 \%, 7 / 18)$, kidney $(27.77 \%, 5 / 18)$, trachea $(22.2 \%, 4 / 18)$ and joint $(5.5 \%, 1 / 18)$. In case of future outbreaks, veterinarians must collect samples of the cited organs to take to the laboratory for ES2 isolation.

The sequences of 16S rRNA from the six strains of ES2 are shown in Table 2. Studied isolates had similarity from 99 to $100 \%$ with those already deposited strains in the GenBank database. Although 16S rRNA gene sequencing is widely used for bacterial identification, the technique has already been described as having low phylogenetic inference at the species level and low discriminatory power for some genus (Mignard and Flandrois 2006). The difficulty to engage in phylogenetically distinct species and/or genus relies on the fact that there are still a low number of sequences deposited in 16S rRNA sequence database. Therefore, more sequences are needed to reduce ambiguity and increase phylogenetic power of ES2 sequences.

Hence, the use of the 16S rRNA region sequencing for microbial identification should be applied with caution, and it should also bring together a harmonious set of guidelines for the sequence interpretation, so that the results of a study could be compared with precision with other ones (Janda and Abbott, 2007). Future studies should be performed using whole genome sequencing of ES2 to verify whether further evaluation of other regions is required to discriminate from different species.

Twenty-eight isolates of ES2 were analysed for antimicrobial resistance. The tested isolates showed complete resistance to antimicrobial agents from the aminoglycoside group (e.g. neomycin and apramycin); a high degree of resistance for 
Table 2 ES2 strains and its $16 \mathrm{~S}$ rRNA sequence along with the comparison with GenBank database

\begin{tabular}{lllll}
\hline Strain & Identification & Size (bp) & $\begin{array}{l}\text { Accession number of the } \\
\text { homologous strain in GenBank }\end{array}$ & \% Homology \\
\hline 1 & F: Erysipelothrix tonsillarum & 1138 & NR_113036.1 & $99 \%$ \\
& R: Erysipelothrix rhusiopathiae & 905 & KP063151.1 & $99 \%$ \\
2 & F: Erysipelothrix rhusiopathiae & 1263 & CP005079.1 & $99 \%$ \\
3 & R: Erysipelothrix rhusiopathiae & 920 & KP063151.1 & $99 \%$ \\
& F: Erysipelothrix rhusiopathiae & 785 & CP005079.1 & $99 \%$ \\
4 & R: Erysipelothrix rhusiopathiae & 911 & KP063151.1 & $100 \%$ \\
& F: Erysipelothrix rhusiopathiae & 1167 & CP005079.1 & $99 \%$ \\
5 & R: Erysipelothrix rhusiopathiae & 914 & KP063151.1 & $100 \%$ \\
& F: Erysipelothrix tonsillarum & 1207 & NR_113036.1 & $99 \%$ \\
6 & R: Erysipelothrix rhusiopathiae & 1247 & KP063151.1 & $99 \%$ \\
& F: Erysipelothrix tonsillarum & 1185 & NR_113036.1 & $99 \%$ \\
& R: Erysipelothrix rhusiopathiae & 922 & CP005079.1 & $99 \%$ \\
\hline
\end{tabular}

$F$ forward, $R$ reverse trimethoprim-sulfamethoxazole $(82.2 \%)$ and fosfomycin (60.7\%); and intermediate or low resistance for tetracycline $(25 \%)$, ceftiofur $(10.7 \%)$, enrofloxacin $(7.1 \%)$, oxytetracycline $(3.6 \%)$, amoxicillin $(3.6 \%)$, norfloxacin $(3.6 \%)$ and lincomycin-spectinomycin (3.6\%). The analysis of antibiotic action sites showed that $67.85 \%$ of the isolates were resistant to at least one of the cell-wall-acting antimicrobials (ceftiofur, amoxicillin and fosfomycin), decreasing to $10.71 \%$ if fosfomycin was excluded. When considering the antimicrobials that inhibited nucleic acid synthesis, $7.14 \%$ of the isolates were resistant to at least one of the antimicrobials (enrofloxacin and norfloxacin). As for the antimicrobials that block protein synthesis, $28.57 \%$ were resistant to at least one of the antimicrobials (tetracycline, oxytetracycline and lincomycin-spectinomycin), excluding apramycin and neomycin, which failed to inhibit bacterial growth in all cases. A total of $82.1 \%$ were resistant to trimethoprim-sulfamethoxazole, which targets folate synthesis. All isolates were resistant to at least one antimicrobial agent, and 18 isolates (64.28\%) were resistant to 3 or more classes of antimicrobial agents and were thus considered multidrug resistant. Eight isolates (28.57\%) were only resistant to two antimicrobial classes.

High resistance to the aminoglycoside group was already expected, since other researches have showed that E. rhusiopathiae was resistant to neomycin (Opriessnig et al. 2004; Balootaki et al., 2017) and apramycin (Opriessnig et al. 2004) and thus these antibiotics may be important to characterize and be used in selective media for this bacterium. The indiscriminate use of broad-spectrum antimicrobials in the poultry industry could have led to the high resistance to trimethoprim-sulfamethoxazole and fosfomycin found in this study. Although still low, resistance to ceftiofur, a thirdgeneration cephalosporin, has high concern due to the importance of third and fourth cephalosporins in the human medicine, and the presence of ESBL (extended-spectrum betalactamases) genes should be further investigated.
Since the classification of the Erysipelothrix genus into six separated species, ES2 had not been isolated from outbreaks of erysipelas in poultry. Before the classification of ES2 as a novel species, it has been classified as E. rhusiopathiae serotype 18 . Its pathogenicity was demonstrated in an experimental test with all 23 serotypes of E. rhusiopathiae, with E. rhusiopathiae serotype 18 inducing local urticarial lesions in swine and being highly virulent to mice (Takahashi et al. 1992).

So far, most of the studies have stated that only E. rhusiopathiae causes erysipelas in poultry, but in the present study, we isolated ES2 associated with septicemia and increased mortality. Therefore, it might be possible that ES2 dissemination has occurred before this report, but it was not correctly identified due to misleading biochemical analysis. In conclusion, the results of the present study demonstrated for the first time ES2 causing disease and mortality in turkeys and high index of isolated antimicrobial resistance. Besides, we found ES2 16S rRNA sequence to be very similar from those of E. rhusiopathiae and E. tonsillarum. We suggest other studies to be conducted to evaluate the possible public health issue due to ES2 incidence in turkey-producing farms.

Funding Conselho Nacional de Desenvolvimento Científico e Tecnológico (CNPq), Fundação de Amparo à Pesquisa do Estado de Minas Gerais (FAPEMIG) and Coordenação de Aperfeiçoamento de Pessoal de Nível Superior (CAPES) for their financial support.

\section{Compliance with ethical standards}

Conflicts of interest The authors declare that they have no conflict of interests.

Research involving human animals The necropsied birds of this work were naturally and recently dead, that by Brazilian Law No. 11,794 of October 8, 2008, there is no need for an evaluation by the Ethics Committee in animal research (because it deals with the handling of dead animals). Even so, we have communicated the Committee on Ethics in the Use of Animals of the Federal University of Uberlandia that certified our work with the number A004/19. 


\section{References}

Ahrné S, Stenstrom I, Jensen NE, Petterson B, Uhlen M, Molin G (1995) Classification of Erysipelothrix strains on the basis of restriction fragment length polymorphisms. Int J Syst Bacteriol 45:382-385

Balootaki PA, Amin M, Haghparasti F, Rokhbakhsh-Zamin F (2017) Isolation and detection of Erysipelothrix rhusiopathiae and its distribution in humans and animals by phenotypical and molecular methods in Ahvaz-Iran in 2015. Iran J Med Sci 42:377-383

Bang BH, Rhee MS, Chang HD, Park DS, Kim BC (2016) Erratum to: Erysipelothrix larvae sp. nov., isolated from the larval gut of the rhinoceros beetle, Trypoxylus dichotomus (Coleoptera: Scarabaeidae). Antonie Van Leeuwenhoek 109:167-168

Bricker JM, Saif YM (2013) Erysipelas. In: Swayne DE, Glisson JR, LR MD, Nolan LK, Suarez LD, Nair V (eds) Diseases of poultry. Iowa State University Press, Ames, pp 812-826

Chooromoney KN, Hampson DI, Eamens GJ, Turner MJ (1994) Analysis of E.rhusiopathiae and E.tonsillarum by multilocus enzyme electrophoresis. J Clin Microbiol 32:371-376

Cohen AL, Fridkin SK, Huang SS, Jernigan JA, Lautenbach E, Oriola S, Ramsey KM, Salgado CD, Weinstein RA (2008) Recommendations for metrics for multidrug-resistant organisms in healthcare settings: SHEA/HICPAC position paper. Infect Control Hosp Epidemiol 29: 901-913

Ikuta NDE, Oliveira SOLLA, Sobral F, Lehmann FK, Da Silveira PV, De Carli S, Casanova YS, Celmer AJ, Fonseca AS, Lunge VR (2014) Taqman real-time PCR assays for rapid detection of avian pathogenic Escherichia coli isolates. Avian Dis 58:628-631

Janda JM, Abbott (2007) 16S rRNA gene sequencing for bacterial identification in the diagnostic laboratory: pluses, perils, and pitfalls. J Clin Microbiol 45:2761-2764
Mignard S, Flandrois JP (2006) 16S rRNA sequencing in routine bacterial identification: a 30-month experiment. J Microbiol Methods 65: 574-581

Opriessnig T, Hoffman LJ, Harris DL, Gaul SB, Halbur PG (2004) Erysipelothrix rhusiopathiae: genetic characterization of Midwest US isolates and live commercial vaccines using pulsed-field gel electrophoresis. J Vet Diagn Investig 16:101-107

Pal N, Bender JS, Opriessning T (2010) Rapid detection and differentiation of Erysipelothrix spp. by a novel multiplex real-time PCR assay. J Appl Microbiol 108:1083-1093

Schmitt F, Schade B, Böhm B, Shimoji Y, Pfahler C (2014) Erysipelas in a free-range flock with conjunctival oedema as an unusual clinical sign. Berl Munch Tierarztl Wochenschr 127:183-187

Takahashi T, Fujisawa T, Tamura Y, Suzuki S, Muramatsu M, Sawada T, Benno Y, Mitsuoka T (1992) DNA relatedness among Erysipelothrix rhusiopathiae strains representing all twenty-three serovars and Erysiplothrix tonsillarum. Int J Syst Bacteriol 42: $469-473$

Takeshi K, Makino S, Ikeda T, Takada N, Nakashiro A, Nakanishi K, Oguma K, Katoh Y, Sunagawa H, Ohyama T (1999) Direct and rapid detection by PCR of Erysipelothrix sp. DNAs prepared from bacterial strains and animal tissues. J Clin Microbiol 37:4093-4098

Verbarg S (2004) Erysipelothrix inopinata sp. nov., isolated in the course of sterile filtration of vegetable peptone broth, and description of Erysipelotrichaceae fam. Nov. Int J Syst Evol Microbiol 54:221-225

Zavalla LD, Swayne DE, Glisson JR, Pearson JE, Reed WM, Jackwood MW (2008) A laboratory manual for the isolation, identification and characterisation of avian pathogens. American association of avian Pathologistis, Washington, D.C, 3:10-11

Publisher's note Springer Nature remains neutral with regard to jurisdictional claims in published maps and institutional affiliations. 